\title{
Improving documentation and use of ultrasound for suprapubic catheter insertions.
}

Nigel Day

\begin{abstract}
The British Association of Urological Surgeons (BAUS) published recommendations for the safe insertion of suprapubic catheters (SPCs) in January 2011. They identified a number of parameters surrounding the insertion and maintenance of catheters that should be identified, implemented and documented on each insertion. One such recommendation was the use of ultrasound, if available and appropriate, to aid insertion and improve patient safety. A previous review of our documentation showed that in 2009 , ultrasound imaging was not utilised in any of the 19 SPC insertions.
\end{abstract}

We retrospectively collected data for 36 SPC insertions between April 2010 and December 2011. The information collated was the same as that collected for the BAUS national SPC insertion audit. This quality improvement project identified a significant increase in the use of ultrasound during SPC insertion since 2009 with 17 out of the 36 (47\%) cases using this imaging modality. Despite this increase, there was still substantial room for improvement. We also identified some areas of documentation that needed improving, particularly with regard to insertion technique and indication for insertion. As a result, a proforma was designed to be used on each SPC insertion ensuring that all required data is easily identifiable and clearly documented. This proforma is now being used as standard mandatory clinical practice within our Trust.

\section{Problem}

The British Association of Urological Surgeons released guidelines in January 2011 with regard to the safe insertion of SPCs. These included good documentation of the procedure with attention paid specifically to the indication for insertion, safe insertion and subsequent catheter care. In our hospital we wanted to investigate whether or not we were compliant with these guidelines and if not, whether we could improve our system to improve patient safety.

\section{Background}

We accessed the online guidelines published by BAUS in January 2011. The BAUS guidelines state the following: 'The suprapubic catheter (SPC) is a useful and widely used tool in urological practice. However, complications can arise from its insertion or ongoing care. Currently there are no guidelines relating to SPC usage. Guidelines are suggested to help maximise safety and ensure best practice in relation to SPC usage.'

The BAUS guidelines looked into all available evidence at the time relating to SPC insertion and if evidence was not available then expert opinions were sought to clarify details.

\section{Baseline Measurement}

We were able to access figures collected in 2009 within the same department at Kingston Hospital which showed that 19 patients had suprapubic catheters inserted. Of these 19 , no cases used ultrasound scan (USS) to aid insertion. Since then use of USS has become part of the guidelines and this was one of the parameters that we looked at.

\section{Design}

Deciding to insert a suprapubic catheter is a relatively straightforward clinical decision. However accurate documentation of the procedure, including method of insertion, assistance to insertion, and indication for insertion is important but not always completed.

By comparing our patient records with the BAUS guidelines we could look at the areas where we fall short of the recommended standards. Also, by producing a proforma with headings for each of the various areas of required documentation, we could bring ourselves up to the required standard, and also have information readily available for future audits.

\section{Strategy}

By designing the proforma we realised that their were a number of different areas to improve on. This would involve educating the doctors and nurses on the wards, and, discussion with the ward, theatre and A\&E managers. Our proposed proforma would have to be well advertised among the doctors and would have to be readily available in all the necessary locations. This would involve liaising with the respective managers to ensure that their was a ready stock of the proformas available. By making it routine to fill in the proformas, there would be a greater chance for the change to 
become 'the norm.'

We discussed the proposed proforma with the clinical governance lead in the department and the prososal was carried forward into the senior management meetings where it was approved. We also presented the proposal at a regional urology meeting where it received a positive response.

\section{See supplementary file: PDSA Cycles2.doc}

\section{Post-Measurement}

We looked at all the criteria set out in the national guidelines. Our audit department helped us to identify all of the new suprapubic catheter insertions performed. We identified 36 supra-pubic catheter insertions during a time period from April 2010 to December 2011 after the guidelines were published. By looking through the notes, we analysed various parameters including the use of ultrasound to aid insertion. There were 12 emergency insertions and 24 elective insertions during the time period. In 17/36 $(47.2 \%)$ insertions, ultrasound was used which was a significant improvement. Since then we have created our proforma which is mandatory for clinicians to complete when inserting SPC. We will be auditing documentation and use of USS in future to see if there has been an improvement, however as the proforma is mandatory we expect $100 \%$ concordance with guidelines.

See supplementary file: userfiles-suprapubic catheters.doc

\section{Lessons and Limitations}

There were several limiting factors identified when carrying out this project. To access the data we relied on the coding department to have correctly identified every patient who underwent a suprapubic catheter insertion. Some patients may have been missed as a result of inaccurate coding. Although we thoroughly went through each set of notes on more than one occasion, sometimes we could not find the relevant piece of documentation. This is not to say it was never documented at all, but it does highlight the need for careful and clear documentation for procedures.

This project would be easy one to undertake at other hospitals.

\section{Conclusion}

We started with an issue regarding procedural documentation within a hospital. This is important for both safety and for medico-legal purposes. Our initial enquiries showed that our hospital was documenting the SPC insertion procedure but that improvements could be made. Once the national guidelines were introduced our documentation improved considerably to allow for a more careful analysis of whether or not we were complying with the guidelines. Even though the standards had improved, there were still a number of shortcomings in documentation, particularly with regard to reasons for not using ultrasound scan assistance when inserting the catheter. By designing a proforma, all of the necessary information could be neatly collated in one place allowing for both accurate description of the procedure as well as allowing us to keep a careful record of the circumstances surrounding that insertion.

We believe this project will improve our clinical practice and also provide a useful tool for early identification of problems in the future. We have discussed the use of the proforma with the relevant areas of the hospital. These included the wards, theatres and $A \& E$ department. Ensuring a steady supply of the proformas to the relevant areas is not always easy, particularly as this procedure is not performed all that frequently in our hospital. The proforma was discussed at the clinical governance meeting for the urology department and agreed upon. We would hope that in future, we will see a further improvement in our documentation to a $100 \%$ compliance record with the national guidelines.

\section{References}

1. Harrison SCW, Lawrence WT, Morley R, Pearce I and Taylor J. British Association of Urological Surgeonsâtu suprapubic catheter practice guidelines. BJU Int 2011; 107(1): 77-85

Acknowledgements: J Schneeweiss, T Nitkunan, RO Morley for their assistance with data collection. 